Fisioter Bras 2017;18(6):727-33

\title{
ARTIGO ORIGINAL \\ Efeito agudo do alongamento por Facilitação Neuromuscular Proprioceptiva associado a TENS e crioterapia na flexibilidade dos músculos isquiossurais de mulheres saudáveis Acute effect of Proprioceptive Neuromuscular Facilitation stretching associated with TENS and cryotherapy in the flexibility of hamstring muscles of healthy women
}

Francisco Roberto Rebouças Junior*, Luana Priscila Diniz Martins*, Moisés Costa do Couto, Ft.,M.Sc. ${ }^{* *}$

*Graduado em Fisioterapia, Especialista em Ortopedia e Traumatologia, Universidade Potiguar, ${ }^{* *}$ Especialista em Biomecânica, Universidade Potiguar, Faculdade Diocesana de Mossoró

Recebido em 10 de julho de 2017; aceito em 23 de outubro de 2017.

Endereço para correspondência: Moisés Costa do Couto, Av. João da Escóssia, 1561, Nova Betânia, 59607-330 Mossoró RN, E-mail: moisescouto@gmail.com, Luana Priscila Diniz Martins: luanaprisdm@gmail.com; Francisco Roberto Rebouças Junior: jnior.roberto@gmail.com

\section{Resumo}

Introdução: O alongamento por Facilitação Neuromuscular Proprioceptiva (FNP) é o mais efetivo dentre os tipos de alongamento. O ganho de flexibilidade após o alongamento se deve também ao aumento da tolerância do indivíduo ao estiramento do músculo, portanto, recursos analgésicos associados ao alongamento por FNP podem potencializar o efeito desta técnica. Objetivo: Verificar o efeito agudo do alongamento por FNP associado a Estimulação Elétrica Nervosa Transcutânea (TENS) e a crioterapia na flexibilidade dos músculos isquiossurais de mulheres saudáveis. Material e métodos: Sessenta voluntarias foram distribuídas aleatoriamente em quatro grupos: (G1) Grupo Controle; (G2) Alongamento por FNP isolado; (G3) Alongamento FNP associado a TENS; (G4) Alongamento FNP associado à crioterapia. Resultados: $\mathrm{Na}$ análise intragrupo foi observado aumento da flexibilidade na comparação antes e depois da aplicação das técnicas em todos os grupos ( $p<0,001)$, exceto no $G 1$. Na análise intergrupo, foi observada diferença estatística apenas na comparação dos três grupos experimentais (G2, G3 e G4) com o grupo controle (G1) ( $p \leq 0,002)$. Conclusão: $O$ alongamento por FNP aplicado isoladamente e associado a TENS ou crioterapia são igualmente eficazes para aumentar a flexibilidade de forma aguda dos músculos isquiossurais de mulheres saudáveis.

Palavras-chave: exercícios de alongamento muscular, propriocepção, analgesia, músculos isquiotibiais.

\begin{abstract}
Introduction: Stretching by Proprioceptive Neuromuscular Facilitation (PNF) is the most effective among the types of stretching. Increased flexibility after stretching is also due to increased tolerance of the subject to the stretching of the muscle, therefore, analgesic resources associated with PNF stretching may potentiate the effect of this technique. Objective: To measure the acute effect of PNF stretching associated with Transcutaneous Electrical Nerve Stimulation (TENS) and cryotherapy in the flexibility of hamstring muscles of healthy women. Methods: Sixty volunteers were randomly assigned to four groups: (G1) Control Group; (G2) PNF Stretching isolated; (G3) PNF with TENS; (G4) PNF associated with cryotherapy. Results: In the intragroup analysis, an increase in flexibility was observed in the comparison before and after the application of the techniques in all groups ( $p<0.001)$, except G1. In the intergroup analysis, a statistical difference was observed only in the comparison of the three experimental groups (G2, G3 and G4) with the control group (G1) ( $p \leq 0.002$ ). Conclusion: PNF stretching applied isolated and associated with TENS or cryotherapy are equally effective to increase the acute flexibility of the hamstring muscles of healthy sedentary women.
\end{abstract}

Key-words: muscle stretching exercise, proprioception, analgesia, hamstring muscles. 
A flexibilidade é a capacidade de mover uma ou várias articulações por toda a amplitude de movimento (ADM) de forma indolor e sem restrições. A flexibilidade é importante por fornecer postura saudável, manter a ADM em um nível satisfatório para a realização de atividades de vida diária ou desportivas, prevenir lesões e melhorar o desempenho físico [1,2]. Sedentarismo, imobilismo, senilidade, distúrbios metabólicos e obesidade são alguns fatores que contribuem para a diminuição de flexibilidade e consequente diminuição da ADM [3-5]. Exercícios de alongamento muscular são comumente utilizados para aumentar a flexibilidade através do estiramento máximo dos músculos [6].

Existem diferentes tipos de alongamento: estático, balístico e por Facilitação Neuromuscular Proprioceptiva (FNP), sendo este último o mais efetivo no ganho de ADM [7-10]. O alongamento por FNP, que também pode ser definido como contrai-relaxa, é caracterizado pela utilização de um alongamento estático, seguido imediatamente de uma contração isométrica máxima do músculo alongado e novamente o alongamento [11]. A justificativa fisiológica para a efetividade deste tipo de alongamento tem sido amplamente discutida na literatura científica, fatores como inibição autógena, redução da viscoelasticidade e principalmente o aumento da tolerância do indivíduo ao alongamento são os mais aceitos [1214].

Sugere-se que este tipo de alongamento seja capaz de gerar um aumento no limiar da dor durante sua realização, fazendo com que parte do ganho de flexibilidade, percebido após o alongamento, se deva ao prolongamento da tolerância ao estiramento [15]. A Estimulação Elétrica Nervosa Transcutânea (TENS) é um recurso que promove analgesia e, portanto, pode aumentar ainda mais a tolerância dolorosa do indivíduo durante o alongamento [16]. A TENS produz um tipo de estimulação tátil capaz de diminuir a sensação dolorosa local, no segmento medular correspondente ao dermátomo estimulado pela corrente elétrica [17], sua ação é explicada pela teoria da comporta da dor [18].

Outros estudos indicam que o uso de recursos que limitem a descarga neural pode diminuir a ativação muscular reflexa, como também a dor, aumentando o efeito de tolerância às manobras de alongamento. Algumas pesquisas têm demonstrado a eficiência da aplicabilidade do gelo com esse propósito, por causar atenuação da atividade do fuso muscular e menor percepção dolorosa [19,20]. Portanto, este recurso associado ao alongamento por FNP também pode potencializar o efeito da técnica.

Não foram encontrados até a presente data, estudos científicos que tenham comparado os efeitos agudos da associação entre a TENS, crioterapia e o alongamento por FNP em um único trabalho. Diante do exposto, este estudo tem como objetivo verificar o efeito agudo do alongamento por FNP associado a TENS (Estimulação Elétrica Nervosa Transcutânea) e a crioterapia na flexibilidade dos músculos isquiossurais de mulheres saudáveis.

\section{Material e métodos}

Tipo do estudo, local e amostra

O presente estudo é um ensaio clínico controlado realizado no Laboratório de Ortopedia e Traumatologia da Universidade Potiguar (UnP) Campus Mossoró/RN. Foram convidadas sessenta voluntárias alunas da universidade para participar do estudo. Após o convite, e assinatura do TCLE (Termo de Consentimento Livre e Esclarecido) as voluntárias responderam uma ficha de avaliação, elaborada pelos pesquisadores, e o Questionário Internacional de Atividade Física (IPAQ), versão curta, para avaliar o nível de atividade física. Após este procedimento, os dados foram analisados e feito a inclusão e exclusão das voluntárias.

\section{Critérios de inclusão e exclusão}

Foram considerados critérios de inclusão: ter idade entre 18 e 30 anos; não fazer uso de medicação analgésica ou relaxante muscular nas últimas $72 \mathrm{~h}$ antes da avaliação; ter Índice de Massa Corporal (IMC) entre 18,5 e 29,9 caracterizado como peso ideal e sobrepeso segundo a OMS (Organização Mundial de Saúde) e ser classificada como sedentária ou irregularmente ativa segundo o IPAQ. Optou-se por não incluir as mulheres consideradas 
ativas ou muito ativas com o objetivo de manter a homogeneidade da amostra, por entender que o nível de flexibilidade dessas mulheres difere de sedentárias e/ou irregularmente ativas.

Foram excluídas as voluntárias que tinham reação alérgica ao gelo; que possuíram hiperflexibilidade, ou seja, flexibilidade $>30 \mathrm{~cm}$ avaliada pelo Banco de Wells; e que apresentassem algum tipo de lesão ortopédica nos membros inferiores.

As voluntárias selecionadas foram distribuídas aletoriamente, através de um site de randomização (www.random.org), em quatro grupos: (G1) grupo controle que não foi submetido a nenhum tipo de intervenção; (G2) grupo submetido ao alongamento por FNP isolado; (G3) grupo submetido ao alongamento por FNP associado a TENS; (G4) grupo submetido ao alongamento por FNP associado à crioterapia. Todos os grupos foram submetidos a avaliação e reavaliação da flexibilidade através do Banco de Wells.

\section{Aspectos éticos}

O estudo foi submetido para a apreciação do Comité de Ética da Universidade Potiguar-UnP sob número CAAE: 59750416.4.0000.5296 e aprovado com o número de protocolo: 1.755.717. O trabalho foi realizado de acordo a Resolução $\mathrm{n} .-466 / 12$ do Conselho Nacional de Saúde. Todas as voluntárias foram informadas dos objetivos do estudo, leram e assinaram o Termo de Consentimento Livre e Esclarecido (TCLE).

\section{Procedimentos}

$\mathrm{O}$ estudo teve três momentos. As participantes foram submetidas à mensuração da flexibilidade dos músculos isquiossurais pelo Banco de Wells (1ำmento), em seguida 0 protocolo de alongamento foi aplicado de maneira isolada, em associação com a TENS ou em associação com crioterapia ( $2^{\circ}$ momento), e então as participantes foram reavaliadas imediatamente após a aplicação dos protocolos para verificação do efeito agudo dos recursos (3ํmomento).

Para a avaliação e reavaliação da flexibilidade dos músculos isquiossurais foi utilizado um Banco de Wells da marca Sanny ${ }^{\circledR}$. Durante as avaliações, as participantes foram posicionadas sentadas sobre um colchonete, os membros inferiores permaneceram em flexão do quadril e extensão de joelho e com as mãos sobrepostas, após o comando dos pesquisadores as voluntárias moveram o escalímetro do banco por meio de uma flexão do tronco até o máximo que lhe fosse possível, mantendo extensão de joelhos, cotovelos e punhos. Foram realizadas três mensurações, das quais foi registrado e analisado o máximo valor encontrado, expresso em centímetros $(\mathrm{cm})$.

O G1 realizou apenas a avaliação através do Banco de Wells descrita anteriormente e, após 25 minutos, a reavaliação. $\mathrm{Na}$ intervenção, o G2 realizou alongamento por FNP de maneira isolada. Para isto, a voluntária ficou em decúbito dorsal, com a coxa contralateral ao membro alongando estabilizada pelo pesquisador. As voluntárias foram instruídas a relaxar totalmente os membros inferiores para não oferecer nenhuma resistência. O pesquisador flexionou passivamente o quadril da participante com joelho estendido até a posição em que a mesma referisse algum desconforto na parte posterior da coxa, em seguida apoiou o mesmo membro inferior da indivídua em seu ombro. Foi solicitado que a voluntária realizasse uma força máxima para estender o quadril por cinco segundos de maneira isométrica contra resistida. Ao final deste tempo, a voluntária relaxou e em seguida teve novamente o quadril flexionado passivamente, até referir um novo desconforto. A manobra foi repetida por duas series de três repetições, mantendo um intervalo de 30 segundos de descanso entre cada uma [21]. Optou-se por manter a contração durante cinco segundos pois é a duração ótima da contração isométrica para a técnica de FNP [22]. O mesmo procedimento foi repetido no membro contralateral.

Para o G3, foi aplicada a TENS convencional da marca Quark, eletrodos quadrados de borracha posicionados de forma coplanar no vente muscular (terço médio) dos músculos isquiossurais. Foram utilizados os seguintes parâmetros: Frequência $100 \mathrm{~Hz}$; duração do pulso $100 \mu$ s e intensidade no limite sensorial tolerável de cada participante, sem contração muscular. A estimulação durou 25 minutos, logo em seguida, os eletrodos foram retirados, feito a higienização do local e realizado o alongamento por FNP, da mesma forma descrita para o G2.

No G4, foi aplicada uma compressa em gel crioterápica da RMC Gel Clínico® de $500 \mathrm{~g}$ congelada sobre um papel toalha no vente muscular (terço médio) dos músculos isquiossurais 
durante 25 minutos. Imediatamente após, foi realizado o alongamento por FNP sob a mesma forma descrita anteriormente.

\section{Análise estatística}

Os dados foram tabulados sob forma de média e desvio padrão, em seguida foi aplicado o teste de Kolmogorov-Smirnov e constatada a normalidade. Na comparação de flexibilidade antes e depois de um mesmo grupo (intragrupo) foi aplicado o teste t pareado para amostras paramétricas. Na comparação do ganho entre todos os grupos (intergrupo), ou seja, a subtração entre os valores do antes e depois de flexibilidade foi utilizado o teste de ANOVA (One-way) para amostras paramétricas e o pós-teste de Tukey. Em todos os casos foi considerado o nível de significância de $p<0,05$.

\section{Resultados}

$\mathrm{Na}$ Tabela I estão sintetizados os dados de caracterização da amostra para cada grupo sob a forma de média e desvio padrão.

Tabela I - Dados de caracterização da amostra.

\begin{tabular}{l|llll}
\hline & G1 & G2 & G3 & G4 \\
\hline idade (anos) & $20,87 \pm 1,46$ & $21,87 \pm 1,12$ & $21,67 \pm 1,91$ & $22,27 \pm 3,43$ \\
peso $(\mathbf{k g})$ & $55,16 \pm 6,26$ & $59,30 \pm 6,43$ & $59,65 \pm 10,04$ & $62,96 \pm 9,94$ \\
altura $(\mathbf{m})$ & $1,58 \pm 0,05$ & $1,62 \pm 0,04$ & $1,63 \pm 0,05$ & $1,60 \pm 0,06$ \\
IMC $\left.\mathbf{( k g} / \mathbf{m}^{2}\right)$ & $21,95 \pm 1,98$ & $22,34 \pm 2,11$ & $22,29 \pm 2,75$ & $24,45 \pm 3,50$ \\
\hline
\end{tabular}

O Gráfico 1 apresenta a comparação da flexibilidade em centímetros de cada grupo. Foi observado aumento significativo na flexibilidade em todos os grupos $(p<0,001)$, exceto no G1.

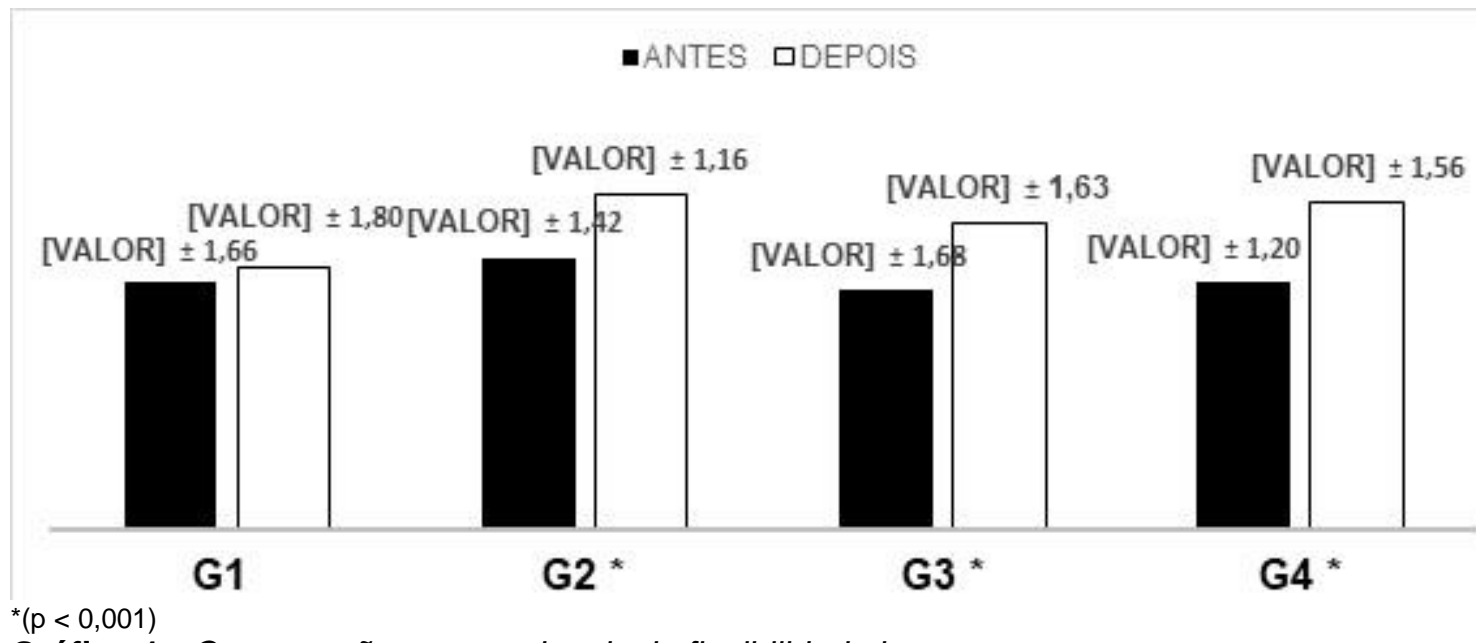

Gráfico 1 - Comparação antes e depois da flexibilidade intragrupo.

O Gráfico 2 mostra a comparação do ganho de flexibilidade intergrupo, ou seja, a diferença de flexibilidade entre a avaliação e reavaliação. É possível identificar que o G2, G3 e o G4 não apresentaram diferença estatística entre si, porém apresentaram aumento de flexibilidade estatisticamente diferente em comparação ao G1. 


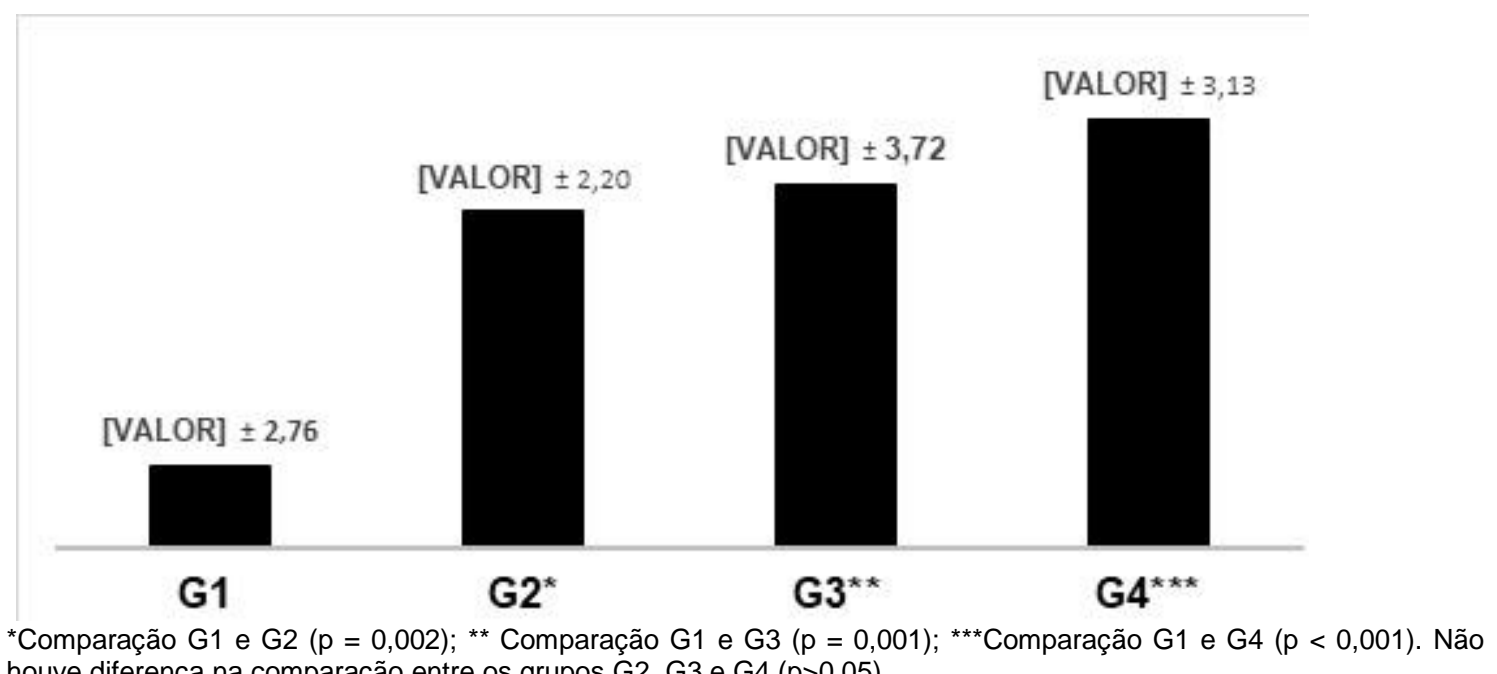

houve diferença na comparação entre os grupos G2, G3 e G4 ( $p>0,05)$.

Gráfico 2 - Comparação do ganho de flexibilidade intergrupo.

Discussão

Os resultados mostraram que os grupos experimentais (G2, G3 e G4) obtiveram aumento da flexibilidade dos músculos isquiossurais através do alongamento por FNP feito de maneira isolada e associada com recursos fisioterapêuticos analgésicos, confirmando sua eficácia neste músculo quanto comparado ao grupo controle (G1).

O G2 realizou o alongamento por FNP de maneira isolada e apresentou aumento de flexibilidade na comparação intragrupo e maior ganho em relação ao G1 (controle). A justificativa da eficácia do alongamento por FNP tem sido bastante discutida na literatura, inicialmente foi proposto que este tipo de alongamento ativa mecanismos neurofisiológicos que desencadeiam a inibição autógena, ou seja, o relaxamento reflexo do músculo alongado pela ativação do Órgão Tendinoso de Golgi (OTG), favorecendo o alongamento do músculo e, portanto, o ganho de flexibilidade. Outras pesquisas contestam a influência da inibição autógena e consideram que alongamento por FNP influencia o ponto em que o estiramento é percebido ou tolerado [13]. A teoria que parece ser mais plausível é a associação destes dois fatores juntamente com a redução da viscoelasticidade que promove o "relaxamento do estresse muscular", diminuindo o torque passivo e a rigidez muscular, mecanismo protetor do músculo; e a inibição recíproca, mas esta só ocorre quando o músculo antagônico ao alongado contrai-se durante a FNP [12,23], o que não foi o caso do presente estudo.

O G3 também apresentou aumento de flexibilidade na comparação intragrupo bem como na comparação do ganho com o G1. A aplicação do alongamento precedido pelo uso da TENS resultou na diminuição da condução dolorosa enquanto o músculo era alongado devido a teoria da comporta da dor $[16,18]$, potencializando o efeito da FNP em aumentar a tolerância da voluntária ao estiramento do músculo [12]. Resultado diferente foi visto por Maciel e Câmara [24] que associaram a TENS com o alongamento estático, e que pode ser justificado pelo fato do alongamento estático talvez não proporcionar 0 aumento da tolerância como comprovadamente a FNP é capaz.

Assim como os demais grupos experimentais, o G4 mostrou aumento de flexibilidade na comparação intragrupo e na comparação com o G1. O alongamento associado à crioterapia promove efeitos que justificam estes ganhos, como a diminuição da atividade futsal. $O$ fuso muscular possui função substancial durante o alongamento do músculo, pois seu impulso facilitatório amplia o grau de tensão do músculo, proporcionando certa limitação de flexibilidade [25], dessa forma, quanto maior for o input sensorial, maior será a ativação muscular. A crioterapia utilizada na presente pesquisa conseguiu atenuar este efeito complicador para 0 alongamento.

Outro efeito da associação entre crioterapia e alongamento por FNP é a analgesia. Conforme descrito anteriormente, a inibição da dor pela ativação de vias nervosas sensitivas cutâneas, aumenta a tolerância da voluntária ao estiramento muscular e o ganho de flexibilidade [12]. Estes resultados são corroborados por estudos anteriores, como o de Brodowicz et al. [26], que afirmam que a aplicação da crioterapia produz maior ganho na flexibilidade do que o alongamento associado ao calor ou ao alongamento estático isolado, 
como também o estudo de Brasileiro et al. [20] que propõe que os efeitos agudos do alongamento são favoráveis quando antecedido por resfriamento através de compressas crioterápicas.

Em contrapartida, tem sido proposto na literatura efeitos contraditórios aos encontrados nesta pesquisa. $O$ resfriamento pode aumentar a rigidez tecidual, reduzindo a flexibilidade e, portanto, diminuindo a eficiência das técnicas de alongamento [27], diante disso, recursos que reduzam a rigidez, como o aquecimento, aumentariam a sua eficácia, conforme evidenciado em outros estudos [28]. Entretanto, este efeito prejudicial não foi visto na presente pesquisa, assim como no estudo de Brasileiro et al. [20], talvez pelo tempo de aplicação menor da crioterapia.

Os resultados mostraram que não há diferença no ganho de flexibilidade entre o alongamento por FNP de maneira isolada e associada com a TENS ou crioterapia, portanto recomenda-se para a prática clínica da fisioterapia a aplicação do alongamento por FNP de maneira isolada, uma vez que este tipo de alongamento por si só já traz ganhos consideráveis. Desta forma, é possível simplificar o procedimento de intervenção, poupar tempo de atendimento, conter gastos financeiros e manter a sensibilidade local como forma de proteção contra o estiramento muscular exagerado. Vale ressaltar que os ganhos de flexibilidade abordados nesta pesquisa são agudos e a persistência destes benefícios carece de avaliações no aspecto longitudinal.

Conclusão

Com os resultados analisados e discutidos no presente estudo, constatou-se que o alongamento por FNP feito isoladamente ou associado aos recursos analgésicos utilizados neste trabalho (TENS ou crioterapia) são igualmente eficazes para aumentar a flexibilidade de forma aguda dos músculos isquiossurais de mulheres saudáveis.

Referências

1. Cyrino ES, Oliveira AR, Leite JC, Porto DB, Dias RMR, Segantin AQ, Mattanó RS, Santos VA. Comportamento da flexibilidade após 10 semanas de treinamento com pesos. Rev Bras Med Esporte 2004;10(4):233-7.

2. Williams DS, Welch LM. Male and female runners demonstrate different sagital plane mechanics as a function of static hamstring flexibility. Braz J Phys Ther 2015;19(5)4218.

3. Farias SH, Maia Neto WL. Atuação da fisioterapia sobre os efeitos do imobilismo no sistema osteomioarticular. Rev Lato Sensu 2008;9(2):47-53.

4. Chang KV, Hung CY, Li CM, Lin YH, Wang TG, Tsai KS et al. Reduced flexibility associated with metabolic syndrome in community-dwelling elders. Plos One 2015;10(1):1-11.

5. Santos ZA, Ribeiro R. Efeito do exercício físico na melhora do grau de flexibilidade na articulação dos joelhos em obesos exercitados comparados com obesos sedentários. Rev Bras Obes Nutr Emag 2016;10(55):20-4.

6. Viveiros L, Polito MD, Simão R, Farinatti P. Respostas agudas imediatas e tardias da flexibilidade na extensão do ombro em relação ao número de séries e duração do alongamento. Rev Bras Med Esporte 2004;10(6):459-63.

7. Sullivan MK, Dejulia JJ, Worrell TW. Effect of pelvic position and stretching method on hamstring muscle flexibility. Med Sci Sport Exerc 1992;24(12):1383-9.

8. Shrier I, Gossal K. Myth and truths of stretching: individualized recommendations for healthy muscles. Phys Sportsmed 2000;28(8):57-63.

9. Spernoga SG, Uhl TL, Arnold BL, Gansneder BM. Duration of maintained hamstring flexibility after a one-time modified hold-relax stretching protocol. J Athl Train 2001;36(1):44-8.

10. Funk DC, Swank AM, Mikla BM, Fagan TA, Farr BK. Impact of prior exercise on hamstring flexibility: a comparison of proprioceptive neuromuscular facilitation and static stretching. J Strength Cond Res 2003;17(3):489-92.

11. Behm DG, Blazevich AJ, Kay AD, McHugh M). Acute effects of muscle stretching on physical performance, range of motion, and injury incidence in healthy active individuals: a systematic review. Appl Physiol Nutr Metab 2016;41(1)1-11. 
12. Hindle KB, Whitcomb TJ, Briggs WO, Hong J. Proprioceptive neuromuscular facilitation (PNF): Its mechanisms and effects on range of motion and muscular function. J Hum Kinet 2012;31:105-13.

13. Chalmers G. Re-examination of the possible role of Golgi tendon organ and muscle splindle reflexes in proprioceptive neuromuscular facilitation muscle stretching. Sports Biomech 2004;3(1):159-83.

14. Nordez A, Cornu C, McNair P. Acute effects of static stretching on passive stiffness of the hamstring muscles calculated using different mathematical models. Clin Biomech 2006;21(7):755-60.

15. Gama ZAS, Medeiros CAS, Dantas AVR, Souza TO. Influência da frequência de alongamento utilizando facilitação neuromuscular proprioceptiva na flexibilidade dos músculos isquiotibiais. Rev Bras Med Esporte 2007;13(1):33-8.

16. Chesterton LS, Barlas P, Foster NE, Lundeberg T, Wright CC, Baxter GD. Sensory stimulation (TENS): effects of parameter manipulation on mechanical pain thresholds in healthy human subjects. Pain 2002;9(1-2):253-62.

17. Campbell JN, Taub A. Local analgesia from percutaneous electrical stimulation. A peripheral mechanism. Arch Neurol 1973;28(5):347-50.

18. Melzack R, Wall PD. Pain mechanisms: a new theory. Science 1965;150(3699):971-9.

19. Kanlayanaphotporn R, Janwantanakul P. Comparison of skin surface temperature during the application of various cryotherapy modalities. Arch Phys Med Rehabil 2005;86(7):1411-5.

20. Brasileiro JS, Faria AF, Queiroz LL. Influência do resfriamento e do aquecimento local na flexibilidade dos músculos isquiotibiais. Rev Bras Fisioter 2007;11(1):57-61.

21. Cornelius WL, Rauschuber MR. The relationship between isometric contraction durations and improvement in acute hip joint flexibility. J Appl Sport Sci Res 1987;1(3):39-41.

22. Bandy WD, Irion JM, Briggler M. The effect of time and frequency of static stretching on flexibility of the hamstring muscles. Phys Ther 1997;77(10):1090-6.

23. Sharman MJ, Cresswell AG, Riek S. Proprioceptive neuromuscular facilitation: mechanisms and clinical implications. Sports Med 2006;36(11):929-39.

24. Maciel ACC, Câmara SMA. Influência da estimulação elétrica nervosa transcutânea (TENS) associada ao alongamento muscular no ganho de flexibilidade. Rev Bras Fisioter 2008;12(5):373-8.

25. Wilmore JH, Costil DL, Kenney WL. Fisiologia do esporte e do exercício. 4⿳亠丷a ed. São Paulo: Manole; 2010.

26. Brodowicz GR, Welsh R, Wallis J. Comparison of stretching with ice, stretching with heat, or stretching alone on hamstring flexibility. J Athl Train 1996;31(4):324-7.

27. Knight KL. Crioterapia no tratamento das lesões desportivas. $1^{\text {a }}$ ed. São Paulo: Manole; 2000.

28. Robertson VJ, Ward AR, Jung P. The effect of heat on tissue extensibility: a comparison of deep and superficial heating. Arch Phys Med Rehabil 2005;86(4):819-25. 